\title{
Practical Adversarial Attacks Against Speaker Recognition Systems
}

\author{
Zhuohang Li \\ The University of Tennessee \\ Knoxville, TN, USA, 37996 \\ zli96@vols.utk.edu \\ Jian Liu \\ The University of Tennessee \\ Knoxville, TN, USA, 37996 \\ jliu@utk.edu
}

\author{
Cong Shi \\ Rutgers University \\ New Brunswick, NJ, USA, 08901 \\ cs1421@winlab.rutgers.edu \\ Bo Yuan \\ Rutgers University \\ New Brunswick, NJ, USA, 08901 \\ bo.yuan@soe.rutgers.edu
}

\author{
Yi Xie \\ Rutgers University \\ New Brunswick, NJ, USA, 08901 \\ yx238@scarletmail.rutgers.edu \\ Yingying Chen \\ Rutgers University \\ New Brunswick, NJ, USA, 08901 \\ yingche@scarletmail.rutgers.edu
}

\begin{abstract}
Unlike other biometric-based user identification methods (e.g., fingerprint and iris), speaker recognition systems can identify individuals relying on their unique voice biometrics without requiring users to be physically present. Therefore, speaker recognition systems have been becoming increasingly popular recently in various domains, such as remote access control, banking services and criminal investigation. In this paper, we study the vulnerability of this kind of systems by launching a practical and systematic adversarial attack against $X$-vector, the state-of-the-art deep neural network (DNN) based speaker recognition system. In particular, by adding a well-crafted inconspicuous noise to the original audio, our attack can fool the speaker recognition system to make false predictions and even force the audio to be recognized as any adversary-desired speaker. Moreover, our attack integrates the estimated room impulse response (RIR) into the adversarial example training process toward practical audio adversarial examples which could remain effective while being played over the air in the physical world. Extensive experiment using a public dataset of 109 speakers shows the effectiveness of our attack with a high attack success rate for both digital attack (98\%) and practical over-the-air attack (50\%).
\end{abstract}

\section{CCS CONCEPTS}

- Security and privacy $\rightarrow$ Software and application security; - Computing methodologies $\rightarrow$ Machine learning; Neural networks.

\section{KEYWORDS}

Speaker Recognition; Deep Learning; Adversarial Example; Room Impulse Response

\section{ACM Reference Format:}

Zhuohang Li, Cong Shi, Yi Xie, Jian Liu, Bo Yuan, and Yingying Chen. 2020. Practical Adversarial Attacks Against Speaker Recognition Systems. In

Permission to make digital or hard copies of all or part of this work for personal or classroom use is granted without fee provided that copies are not made or distributed for profit or commercial advantage and that copies bear this notice and the full citation on the first page. Copyrights for components of this work owned by others than ACM must be honored. Abstracting with credit is permitted. To copy otherwise, or republish, to post on servers or to redistribute to lists, requires prior specific permission and/or a fee. Request permissions from permissions@acm.org.

HotMobile '20, March 3-4, 2020, Austin, TX, USA

(c) 2020 Association for Computing Machinery.

ACM ISBN 978-1-4503-7116-2/20/03...\$15.00

https://doi.org/10.1145/3376897.3377856
Proceedings of the 21st International Workshop on Mobile Computing Systems and Applications (HotMobile '20), March 3-4, 2020, Austin, TX, USA. ACM, New York, NY, USA, 6 pages. https://doi.org/10.1145/3376897.3377856

\section{INTRODUCTION}

Research interest in voice controllable system (VCS) has grown considerably in recent years as the system provides a convenient way of meeting a user's various daily needs through voice commands. With such a convenient and ubiquitous speech interface, speaker recognition system (a.k.a. voice recognition system), identifying the voice of a given utterance among a set of enrolled speakers, could be seamlessly integrated and thus be used to facilitate a series of security-enhanced voice-based applications. For instance, new features in recent Apple Siri can reliably recognize voices, enabling HomePod to respond to requests from multiple users in shared spaces. Some companies (e.g., Voice Biometrics Group [7]) use speaker recognition technologies to let people gain access to information or give authorization without being physically present. Chase Voice ID [2] exploits remote voice authentication to quickly verify users and prevent fraud when they call the bank's customer service center. It has shown a critical need for deploying voiceprint recognition systems on the top of many existing applications.

Existing studies have demonstrated that applying deep neural networks (DNN) to speaker recognition task has great advantages in terms of its highly scalable embedding performance and the ability of coping with practical interference (e.g., noises and reverberation). For instance, DNN posteriors have been used to derive sufficient statistics for alternative i-vectors calculation allowing to discriminate speakers at triphone level [11]. The researchers also showed that DNN-based solutions could lead to a significant improvement over current state-of-the-art solutions, such as conventional universal background model-Gaussian mixture model (UBM-GMM), on telephone speech [13]. However, DNN model has been shown as serious vulnerability when facing intentionally distorted inputs. For instance, adversarial examples could fool the model to make false classifications. Thus, DNN-based speaker recognition systems would be inevitably threatened by the adversarial examples.

In the audio space, Carlini et al. [3] recently demonstrated that by adding an inconspicuous perturbation, an adversary could force the automatic speech recognition (ASR) system to misrecognize a speech command as any adversary-desired text. Moreover, CommanderSong [24] embeds malicious commands into regular songs, 
making people treat them as regular music whereas ASR systems would recognize them as commands and carry them out accordingly. Different from ASR system that mainly focuses on speechto-text translation, speaker recognition model utilizes embedding methods to extract features that represent voice similarities to distinguish speakers regardless of their speech content. To the best our knowledge, the only existing adversarial attack [10] against speaker recognition system targets for an end-to-end speaker verification model, which is a binary speaker recognition system that gives either accept or reject by verifying whether the voice is uttered by a claimed speaker. By adding an inconspicuous perturbation to the original voice, the model might produce incorrect outputs such as rejecting a legitimate user or vice versa. Additionally, such an attack only considers digital scenarios, in which the generated adversarial sample is directly fed into the speaker recognition system without being played through a speaker.

In this paper, we explore the possibility of conducting an overthe-air adversarial attack in practical scenarios, in which the adversarial examples are played through a loudspeaker to compromise speaker recognition devices. Specifically, our testing model is $\mathrm{X}$ vector [18], the state-of-the-art DNN-based multi-class speaker recognition model, with 109 speakers. We show that by adding an inconspicuous perturbation into the original audio, our attack can deceive the speaker recognition system causing a false prediction. To launch such an attack, a few challenges we face include: (1) Unlike binary speaker verification system, attacking a multi-class speaker recognition model requires more sophisticated adversarial learning processes to make the adversarial examples to be classified as the adversary-desired speaker; (2) To make the adversarial examples remain effective while being played over the air, the added perturbation needs to be robust enough to survive real-world distortions caused by different audio propagation channels (e.g., multi-path effect), ambient noises sources, and speaker \& microphone limitations; and (3) The distortion between the generated adversarial example and the original speech should be as small as possible, making the example stealthy and unnoticeable to human.

In particular, we applied gradient-based adversarial machine learning algorithms to generate adversarial examples for two types of representative attacks: (1) Untargeted attack that aims to disable the speaker recognition system by making the audio signals misclassified as incorrect speakers; and (2) Targeted attack that is designed to change the classification result to an adversary-desired speaker, which will enable the adversary to pass the authentication with fraudulent identities. To generate untargeted adversarial examples, we adapt fast gradient sign method (FGSM) [6] to compute the adversarial perturbation by taking the derivative of the cross-entropy loss between the output probability distribution and its true label. Whereas the targeted adversarial examples are computed through solving an optimization process to minimaize the cost function of the noise level as well as the distance to the targeted class. In order to effectively launch an over-the-air adversarial attack to compromise speaker recognition devices, we estimate the room impulse response (RIR) and consider the inferred distortions of the recorded sound at microphone end in the adversarial learning phase. By exploiting the inherent weakness of the deep learning models, the proposed adversarial attack shows several advantages over conventional replay and synthesis attacks: (1) The proposed attack does not require the adversary to have the ability to collect any audio clips from the victim, making it easier to be launched in practice; (2) The proposed attack can make the crafted speech recognized as any adversary-desired speaker within the enrolled set, without requiring the adversary to explicitly collect vocal information from each speaker; (3) By injecting well-crafted inconspicuous perturbations, the proposed attack is able to pass Probabilistic Linear Discriminant Analysis (PLDA) and Factor Analysis (FA) techniques which are widely adopted in modern speaker recognition models and have been proved effective in defending against conventional attacks [22]. Our main contributions are summarized as follows.

- To the best of our knowledge, this is the first work of designing practical and systematic adversarial attack, including both untargeted and targeted attacks, against multi-class speaker recognition system.

- We propose to use the estimated RIR that reflects acoustic channel state information to generate practical audio adversarial examples that can still remain effective while being played over the air in a realistic environment.

- We implement gradient-based adversarial learning algorithms to make the generated adversarial examples unnoticeable to humans and effectively compromise speaker recognition systems.

- Extensive experiments, including both digital attack and practical over-the-air attack, are conducted using a public dataset of 109 English speakers. The results show that our attack achieves a high attack success rate of over $98 \%$ for digital attack and $50 \%$ for over-the-air attack.

\section{RELATED WORK}

Attacks on Speech Recognition Systems. Recent studies have demonstrated the potential of spoofing automatic speech recognition (ASR) systems with adversarial attacks. As an initial study, Carlini and Wagner [3] develop an adversarial attack against DNNbased ASR system. By adding an imperceptible perturbation, an audio waveform could be transcribed as any desired target phrase. However, the adversarial example, i.e., an audio waveform combined with a perturbation, would lose its effectiveness after being played over-the-air. This problem is further investigated in a later work [16], which achieves over-the-air attack at simulated environments. In addition, CommanderSong [24] develops a more practical over-the-air attack which stealthily embeds voice commands into songs through adversarial learning. The songs could then be played from a remote loudspeaker to launch attacks. These attacks target only speech recognition models, while few studies explore the vulnerability of speaker recognition systems to the adversarial attacks.

Attacks on Speaker Recognition Systems. Traditional attacks against speaker recognition systems could be broadly categorized as replay attack [21], speech synthesis attack [12], impersonation attack [1], and voice conversion attack [9]. In particular, replay attack [21] uses pre-recorded voice samples of the user to spoof the speaker recognition system. Such an attack, however, is less effective in many practical scenarios, such as call center where a speaker uses his voice for both authentication and interaction. The inconsistency of the voice could alert the staff and thwart the actual attack (e.g., request bank transfer). To avoid such inconsistencies, speech synthesis attacks [12] generate a victim's voice by learning an acoustic model from a limited set of voice samples. 


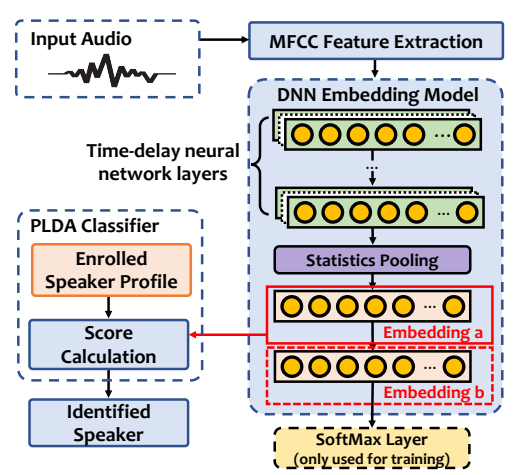

Figure 1: Architecture of X-vector system.

However, human and synthetic speeches could be differentiated with higher order Mel-cepstral coefficient [4], or deep neural netowrk [23], rendering such attacks ineffective. Furthermore, an adversary could imitate a victim's voice through impersonation/voice conversion $[1,9]$ by manipulating existing voice samples from other users. However, these attacks could be defended by PLDA and Factor Analysis (FA) techniques which are usually integrated in state-of-the-art DNN-based speaker recognition models [22]. In comparison, our adversarial attack could circumvent the defense mechanisms while stealthily altering the DNN model's outputs without introducing noticeable distortion of speech. Additionally, different from backdoor attacks [8] which aims to force the ML system to misclassify instances by crafting adversarial primitive learning modules (PLM) at the model training stage, in this paper, we focus on performing adversarial attacks by generating adversarial examples without modifying models. The most related study [10] develops an adversarial attack against an end-to-end DNN-based speaker verification model. This work, however, only considers a simple binary classification problem in the digital field without playing the adversarial examples over the air. Differently, in this paper we are the first to explore the vulnerability of state-of-the-art multi-class speaker recognition systems by developing a practical over-the-air adversarial attack.

\section{SPEAKER RECOGNITION SYSTEM \& THREAT MODEL}

\subsection{Target Speaker Recognition System}

In this work, we choose X-vector architecture [18] as the target speaker recognition system as it shows a superior performance comparing to traditional i-vector models, and has been used as baseline in several follow-up studies [17]. As illustrated in Figure 1, the X-vector system first takes an input audio and divided into frames and extract mel frequency cepstral coefficents (MFCCs) features. The extracted features are then fed into a time-delay neural network (TDNN). At each layer, TDNN computes the activation of frames at the current and neighboring time steps. Subsequently, a statistics pooling layer aggregates the input segment by taking the mean and standard deviation of the output from the last frame-level layer. In addition, two fully-connected layers are used to map the concatenated statistics into embeddings. Finally, the PLDA classifier computes the probability of the voice belonging to each speaker in the enrollment set by comparing the similarity between the embedding $a$ taken from the second last hidden layer and the enrolled

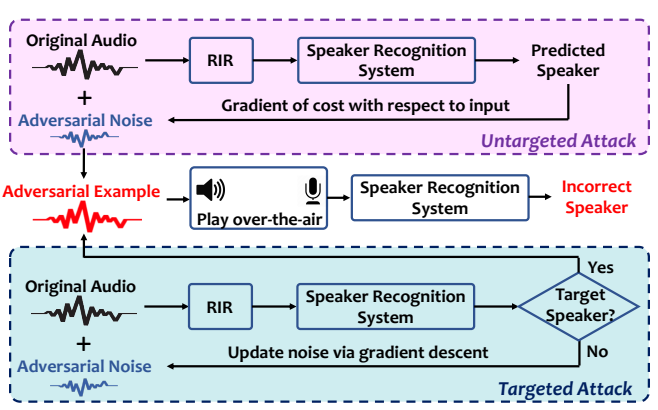

Figure 2: Attack overview.

speaker profile. A prediction is made by choosing the speaker with the highest calculated probability.

Attack Chance on DNN Model. During the training phase, the DNN model leverages gradient descent algorithms to learn the mapping between the input space and the embedding space. However, if the trained model architecture and parameters are known, the gradient information will remain trackable. Therefore, with the direction of the gradient, it is possible for an adversary to add a well-crafted perturbation to the input and consequently manipulate the output prediction. Moreover, by taking advantage of gradient-based algorithms, the computed perturbation can be so subtle as to be unnoticeable to human.

\subsection{Threat Model}

As is used in previous studies on adversarial attacks against speech recognition [3] and speaker verification system [10], we assume a white-box setting, where the adversary has complete knowledge to the speaker recognition model. This is a reasonable assumption in practice as many speaker recognition systems are built upon pretrained speaker recognition models which are typically available online (e.g., a pre-trained x-vector model is offered in Kaldi [15]). The adversary also has access to the physical environment where the actual attack will be launched, and is capable of utilizing a speaker and microphone to measure the room impulse response (RIR) to launch over-the-air attacks. Specifically, the flow of our attack is shown in Figure 2, where the adversarial examples are generated from two types of attacks, and played over-the-air to deceive the speaker recognition system at the microphone side. To make the adversarial examples remain effective while being played in the air, we use the estimated RIR to model the sound distortion when generating adversarial examples. The detailed generation flow is presented as follows:

Untargeted Attack. The input audio signal first goes through the measured RIR to simulate the played over-the-air process. The speaker recognition system then takes the distorted signal and makes a prediction. The untargeted adversarial noise is computed by taking the gradient of the cost function (i.e. multi-class cross entropy) with respect to the input audio. Finally, the adversarial example is constructed by adding the computed noise to the original audio.

Targeted Attack. The targeted attack works in an iterative way: First, the targeted adversarial noise is initialized with zeros. The original audio is then combined with the adversarial noise and modified according to the estimated RIR. A prediction is made by the speaker recognition system. If the prediction does not match 


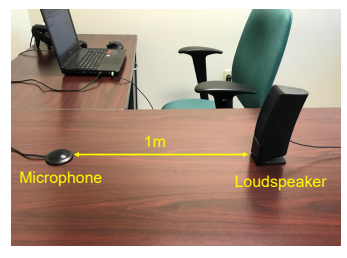

(a) Experimental setup

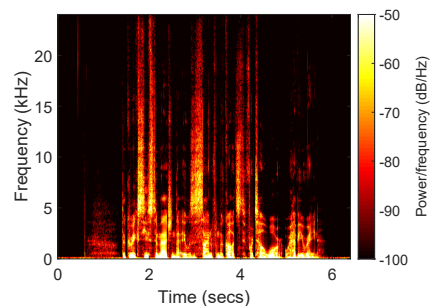

(b) Played voice

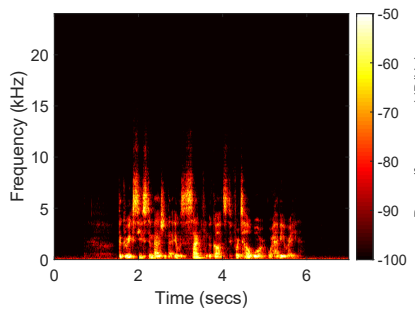

(c) Recorded voice

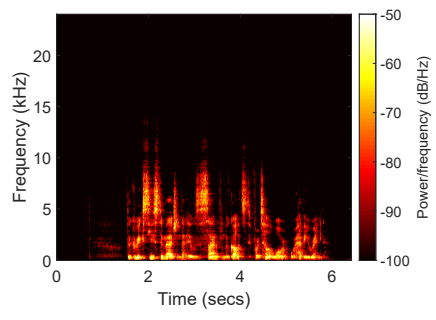

(d) Predicted voice (w/ RIR)

Figure 3: Preliminary experiment to verify the effectiveness of the estimated RIR.

the adversary-desired speaker, the noise will be modified according to the gradient descent's direction where the probability of the target class increases. If the prediction and the adversary-desired speaker are matched, the adversarial example is simply the sum of the original audio and the adversarial noise.

With the generated adversarial example, the attack could be launched in many practical scenarios by using a nearby loudspeaker. For instance, an adversary might attack the voice assistant in shared spaces, making it respond to identity-based service requests with a fraudulent identity; an insider might attack the voiceprint-based security entrance system to gain access to certain building/office to steal sensitive information; an imposter might call a bank's contact center to transfer money to his own account by making himself recognized as the victim.

\section{GENERATING PRACTICAL ADVERSARIAL EXAMPLES}

\subsection{Room Impulse Response Estimation}

Since digital adversarial example would be most likely to lose its effectiveness during the over-the-air process, modeling the sound distortions during audio propagation is crucial for launching practical attacks. We propose to employ RIR [19], which characterizes the preposition of acoustic signals propagating through different paths (i.e., direct path and other reflected paths) with various attenuations and delays. It can be used to model the transfer function between the played voice $x(t)$ and the recorded voice $y(t)$. Specifically, the transfer function of audio propagation can be modeled as:

$$
y(t)=K[x(t)] \otimes h^{\prime}(t)+n(t),
$$

where $K[\cdot]$ is the $N$-th order discrete-time Volterra kernel to represent a nonlinear memoryless system, which is usually used to model harmonic/non-linear distortions caused by the nonlinearity of loudspeaker and microphone. $h(t)^{\prime}$ is an impulse response characterizing linear distortions (i.e., delays and attenuations) and $\otimes$ denotes the convolution process. Additionally, some noises $n(t)$ uncorrelated with the input signal are added to the output. Since it is difficult to separate the responses of $h^{\prime}(t)$ from the $K[\cdot]$ in practice, the sound propagation is simplified as:

$$
y(t)=x(t) \otimes h(t)
$$

where $h(t)$ is the RIR, a composite response to represent both linear and nonlinear characteristics and can be estimated using audio measurement techniques [5].

Specifically, we first play an excitation signal $x_{e}(t)$, where the signal frequency varies exponentially with time and play it through a loudspeaker. The signal allows each harmonic distortion at each order pack into a separate impulse response [5] and can be represented as:

$$
x_{e}(t)=\sin \left(\frac{2 \pi f_{1} T}{\ln \left(\frac{f_{2}}{f_{1}}\right)}\left(e^{\frac{t}{T} \ln \left(\frac{f_{2}}{f_{1}}\right)}-1\right)\right),
$$

where $f_{1}, f_{2}$ are the start and stop frequencies of sweeping, respectively, and $T$ is the signal duration. In our RIR estimation, we set $f_{1}=20 \mathrm{~Hz}, f_{2}=20 \mathrm{kHz}$ and $T=5 \mathrm{~s}$. When being played through a loudspeaker, $x_{e}(t)$ has a constant magnitude and is followed by a few seconds of silence to avoid sound aliasing caused by multi-path effects/harmonic distortions. With the response $y_{e}(t)$ recorded by the microphone, the room impulse response (RIR) $h(t)$ can be estimated by convolving it with an inverse filter: $h(t)=y_{e}(t) \otimes f(t)$, where the filter $f(t)$ is the time-reversal of $x_{e}(t)$.

A preliminary experiment is conducted to verify the effectiveness of the proposed RIR estimation. As shown in Figure 3(a), a loudspeaker and a microphone is placed on a table in a typical office environment. We use the loudspeaker to play a voice human speech sample and record it with the microphone. Figure 3(b) shows the spectrogram of the played voice sample, while Figure 3(c) and 3(d) are the spectrograms of the voice samples recorded by the microphone and predicted one using the estimated RIR, respectively. To quantify the similarity between recorded and predicted voice samples, we use mean square error (MSE) to measure the difference between 2D spectrograms. The MSE between the recorded and predicted voice samples is 0.112 , while the error achieves 0.84 between the played and the recorded voice samples. The high similarity between the recorded and predicted voice samples demonstrate the effectiveness of our RIR estimation.

\subsection{Adversarial Example Generation}

The X-vector system showed in Figure 1 can be viewed as a function $f(\cdot)$, which takes as an input utterance $X$ and outputs a probability vector $P=\left[p_{1}, \ldots, p_{i}\right]$, containing the predicted probability scores $p_{i}$ for each speaker $i$. The untargeted and targeted adversarial examples can be generated in the following adversarial learning processes, respectively.

Untargeted Adversarial Example. It is constructed by adding a perturbation $\delta$ to the original input utterance. To be explicit, we write the adversarial example $X^{\prime}=X+\delta$. Due to the local linearity of DNN models, a linear perturbation is sufficient to be constructed for an untargeted attack (referred as the fast gradient sign method (FGSM) [6]):

$$
\delta=\epsilon \operatorname{sign}\left(\nabla_{X} J(X, y)\right),
$$

where $\operatorname{sign}(\cdot)$ denotes the signum function, $J(X, y)$ represents the cost function between the input $X$ and corresponding label $y$, and $\epsilon$ 
Table 1: Results of digital untargeted attack.

\begin{tabular}{|c|c|c|c|c|c|c|}
\hline Attack Strength (i.e., $\epsilon$ ) & No Attack & $10^{-5}$ & $10^{-4}$ & $10^{-3}$ & $10^{-2}$ & $10^{-1}$ \\
\hline Speaker Recognition Accuracy (\%) & 92.81 & 84.71 & 41.33 & 12.11 & 2.23 & 1.37 \\
\hline Attack Success Rate (\%) & - & 8.73 & 55.47 & 86.95 & 97.60 & 98.52 \\
\hline Average Distortion (dB) & - & -89.06 & -69.15 & -49.24 & -29.33 & -9.41 \\
\hline
\end{tabular}

is a pre-choosen constant to control the attack strength. We use the cross-entropy between the predicted probability vector and the true label as the cost function: $J(X, y)=-y \cdot \log (P)$. For conventional digital attack, the untargeted adversarial example is generated by:

$$
X^{\prime}=X+\epsilon \operatorname{sign}\left(\nabla_{X}(-y \cdot \log (f(X)))\right) \text {. }
$$

To derive practical adversarial examples that remain effective while being played over-the-air, we model the air channel using the estimated RIR $h$. The input audio $X$ is first convolved with the estimated $h$ to simulate the over-the-air process, and the adversarial example can be generated as:

$$
X^{\prime}=X+\epsilon \operatorname{sign}\left(\nabla_{X}(-y \cdot \log (f(X \otimes h)))\right) .
$$

Targeted Adversarial Example. The adversarial example targeting at label $y_{t}$ can be generated through solving an optimization problem:

$$
\text { minimize }\|\delta\|_{2} \text {, s.t. } f(X+\delta)=y_{t},
$$

where $\|\cdot\|_{2}$ denotes the $L_{2}$ norm. As solving the direct non-linear constrained non-convex problem is difficult, in practice, we solve:

$$
\operatorname{minimize}-y_{t} \cdot \log (f(X+\delta))+c\|\delta\|_{2},
$$

where $c$ is a pre-choosen constant which controls the attack strength. Specifically, the first term will be reduced when the predicted distribution is aligning the target label, and the second term penalizes the perturbation magnitude. Gradient descent is applied to find the optimal perturbation $\delta^{*}$, and an targeted adversarial example is generated by $X^{\prime}=X+\delta^{*}$. To be effective under practical settings, the optimization process (i.e., Equation 8) needs to be reformulated to include RIR:

$$
\text { minimize }-y_{t} \cdot \log (f((X+\delta) \otimes h))+c\|\delta\|_{2} .
$$

\section{ATTACK EVALUATION}

\subsection{Experimental Methodology}

Dataset and Basedline Model. We evaluate our attack on the dataset CSTR VCTK Corpus [20], which contains total 44217 utterances spoken by 109 English speakers with various accents. The dataset is splitted into training and testing sets with a ratio of 4 to 1. The MFCC features are 30 dimensional MFCCs and derived with a frame length of $25 \mathrm{~ms}$. A pre-trained X-vector model provided in Kaldi [15] is used for embedding extraction. Regarding the computing environment, a NVIDIA DGX-1 server with $4 \times$ Tesla V100 GPU (32GB memory) is used.

Evaluation Metrics. (1) Speaker Recognition Accuracy: The percentage of utterances being correctly recognized by the baseline model. (2) Attack Success Rate: The ratio of the number of succeeded attacks to the total number of attack attempts. (3) Distortion Metric: We quantify the relative loudness of the introduced perturbation with respect to the original utterance in decibel: $D(\delta, X)=$ $20 \log _{10}\left(\frac{\max (\delta)}{\max (X)}\right)$.

Attack Settings. For the digital untargeted attack, the speaker recognition accuracy on clean dataset is recorded as benchmark and
Table 2: Results of digital targeted attack.

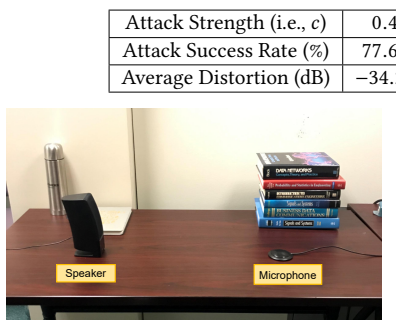

(a) Office

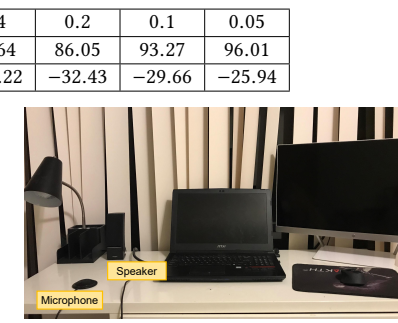

(b) Apartment
Figure 4: Experimental setups for the practical over-the-air adversarial attack.

compared with that on the adversary-perturbed dataset. The digital targeted attack is evaluated by attempting to generate adversarial examples for every speaker to target at all other 108 speakers. The practical attack is tested in two real-world scenarios, as shown in Figure 4, where we play and record the adversarial examples generated by the digital and practical attack, respectively, and compare the attack success rate.

\subsection{Evaluation of Digital Attacks}

Table 1 shows the effectiveness of the untargeted attacks under different $\epsilon$ settings, where a larger $\epsilon$ value could enable stronger attack but lead to more significant distortions. Specifically, the baseline model could achieve $92.81 \%$ speaker recognition accuracy when no attack is present. Under untargeted attacks, the attack success rate grows with the attack strength and reaches $97.6 \%$ when $\epsilon=10^{-2}$, where the baseline model could only correctly recognize $2.23 \%$ of the utterances.

Table 2 presents the results of our digital targeted attack. We report attack success only when the resulting speaker matches the desired targeted speaker. Similar to the observations in untargeted attack, the attack success rate increases with the attack strength (i.e., c). Specifically, when $c=0.2$, our attack can achieve a $86.05 \%$ attack success rate while keeping the average distortion at -32.43 $\mathrm{dB}$, which is approximately the difference between the ambient noise in a quite room and a person talking [3].

\subsection{Evaluation of Practical Attacks}

As shown in Figure 4, for each scenario, we use a loudspeaker to play 10 digital/practical adversarial examples and the voice samples are recorded by the microphone. An untargeted attack is reported success if a speaker is misclassified, while an targeted attack is considered as success only when the recorded voice sample is recognized as the targeted speaker. For untargeted attacks, both digital and practical attacks in the two environments can achieve $100 \%$ attack success rate. This is because the speaker recognition, even for the state-of-the-art X-vector, could be impacted by various environmental interferences (e.g., multipath, ambient noises) and thus mis-classified, which makes the untargeted attack less challenging. Table 3 shows the attack success rates of the targeted attacks through playing digital adversarial examples and practical ones which are generated by integrating RIR into the training process. We can observe that the practical attack can achieve a $50 \%$ attack success rate in both environments, while only one digital adversarial example succeed in spoofing the baseline model in the apartment. 
Table 3: Attack success rate of over-the-air targeted attack.

\begin{tabular}{|c|c|c|}
\hline & $\begin{array}{l}\text { Playing digital } \\
\text { adversarial examples }\end{array}$ & $\begin{array}{l}\text { Playing practical } \\
\text { adversarial examples }\end{array}$ \\
\hline Office & $0 \%$ & $50 \%$ \\
\hline Apartment & $10 \%$ & $50 \%$ \\
\hline
\end{tabular}

This is because the proposed RIR estimation could precisely characterizes the acoustic channel state information, making it possible to launch over-the-air adversarial attacks.

\section{CONCLUSION AND FUTURE WORK}

In this paper, we explore the vulnerability of speaker recognition systems with a practical and systematic adversarial attack against $X$ vector. We apply several gradient-based adversarial machine learning algorithms to generate digital adversarial examples for both untargeted and targeted attacks. In order to design more practical adversarial examples that remain effective when being played over the air, we integrate the estimated room impulse response into the adversarial example generation. Extensive experiment in both digital and real-world settings demonstrate the effectiveness of the proposed attacks.

As for future work, we plan to explore the following directions:

Practical Black-box Attack. To generate adversarial perturbations without prior information (e.g., architecture, parameters) of a target model, we plan to explore the feasibility of generating adversarial examples by leveraging gradient-free optimization algorithms (e.g., genetic algorithm) or training a substitute model. In the scenarios where estimating the exact RIR is infeasible, we will exploit room simulators to approximate the actual RIR and launch a practical attack. We will also evaluate such a practical black-box attack on commercial speaker recognition systems (e.g., Talentedsoft, and Microsoft Azure).

Dynamic Environment \& Far-field Attack. In many practical scenarios in which the loudspeaker is at a far-field distance or the surrounding environment is dynamically changing, the onetime estimated RIR might be not accurate. To cope with this issue, we plan to exploit RIR augmentation techniques to model the variations caused by such complexity. Specifically, we plan to exploit direction-to-reverberant ratio (DRR), which represents the ratio of the total energy pertaining to the sound propagating from the direct path and that from the other reflected paths, to model the RIR variations. A group of possible RIRs can be derived by tuning the DRR of the RIR obtained via physical measurement. By fusing the group of augmented RIRs into the adversarial examples generation process, we could generate more robust adversarial examples that are resilient to dynamic environmental changes as well as the RIR measurement error in far-field cases.

Bypassing Liveness Detection. Adversarial attacks launched by playing through loudspeakers are most likely to be defended by liveness detection mechanisms. A possible way to bypass liveness detection is to devise a type of audio-agnostic universal perturbation [14] that can fool the speaker recognition with arbitrary audio inputs. By injecting such a universal perturbation while a live user is speaking, the adversary might be able to deceive the system armed with liveness detection mechanism.

\section{ACKNOWLEDGMENTS}

We would like to thank our shepherd, Dr. Wei Gao, and our reviewers for their insightful feedback. This research is supported in part by the National Science Foundation grants CNS1820624 and CNS1814590), the Army Research Office grant W911NF-18-1-0221 and the Air Force Research Laboratory grant FA8750-18-2-0058.

\section{REFERENCES}

[1] Talal B Amin, James S German, and Pina Marziliano. 2013. Detecting voice disguise from speech variability: Analysis of three glottal and vocal tract measures. In Proceedings of Meetings on Acoustics 166ASA, Vol. 20.

[2] Chase Bank. 2019. Security as unique as your voice. https://www.chase.com/ personal/voice-biometrics

[3] Nicholas Carlini and David Wagner. 2018. Audio adversarial examples: Targeted attacks on speech-to-text. In IEEE SPW 2018. 1-7.

[4] Lian-Wu Chen, Wu Guo, and Li-Rong Dai. 2010. Speaker verification against synthetic speech. In 2010 7th International Symposium on Chinese Spoken Language Processing. IEEE, 309-312.

[5] Angelo Farina. 2000. Simultaneous measurement of impulse response and distortion with a swept-sine technique. In Audio Engineering Society Convention 108. Audio Engineering Society.

[6] Ian J Goodfellow, Jonathon Shlens, and Christian Szegedy. 2014. Explaining and harnessing adversarial examples. arXiv preprint arXiv:1412.6572 (2014).

[7] Voice Biometrics Group. 2019. Voice Biometrics Group. https://https://www. voicebiogroup.com/

[8] Yujie Ji, Xinyang Zhang, and Ting Wang. 2017. Backdoor attacks against learning systems. In IEEE CNS 2017. 1-9.

[9] Tomi Kinnunen, Zhi-Zheng Wu, Kong Aik Lee, Filip Sedlak, Eng Siong Chng, and Haizhou Li. 2012. Vulnerability of speaker verification systems against voice conversion spoofing attacks: The case of telephone speech. In IEEE ICASSP 2012. 4401-4404.

[10] Felix Kreuk, Yossi Adi, Moustapha Cisse, and Joseph Keshet. 2018. Fooling end-to-end speaker verification with adversarial examples. In IEEE ICASSP 2018. 1962-1966.

[11] Yun Lei, Nicolas Scheffer, Luciana Ferrer, and Mitchell McLaren. 2014. A novel scheme for speaker recognition using a phonetically-aware deep neural network. In IEEE ICASSP 2014. 1695-1699.

[12] Johan Lindberg and Mats Blomberg. 1999. Vulnerability in speaker verification-a study of technical impostor techniques. In Sixth European Conference on Speech Communication and Technology.

[13] Mitchell McLaren, Yun Lei, and Luciana Ferrer. 2015. Advances in deep neural network approaches to speaker recognition. In IEEE ICASSP 2015. 4814-4818.

[14] Seyed-Mohsen Moosavi-Dezfooli, Alhussein Fawzi, Omar Fawzi, and Pascal Frossard. 2017. Universal adversarial perturbations. In Proceedings of the IEEE conference on computer vision and pattern recognition. 1765-1773.

[15] Daniel Povey, Arnab Ghoshal, Gilles Boulianne, Lukas Burget, Ondrej Glembek, Nagendra Goel, Mirko Hannemann, Petr Motlicek, Yanmin Qian, Petr Schwarz, Jan Silovsky, Georg Stemmer, and Karel Vesely. 2011. The Kaldi Speech Recognition Toolkit. In IEEE 2011 Workshop on Automatic Speech Recognition and Understanding. IEEE Signal Processing Society. IEEE Catalog No.: CFP11SRW-USB.

[16] Yao Qin, Nicholas Carlini, Garrison Cottrell, Ian Goodfellow, and Colin Raffel. 2019. Imperceptible, Robust, and Targeted Adversarial Examples for Automatic Speech Recognition. In International Conference on Machine Learning. 5231-5240.

[17] Suwon Shon, Hao Tang, and James Glass. 2018. Frame-level speaker embeddings for text-independent speaker recognition and analysis of end-to-end model. In 2018 IEEE Spoken Language Technology Workshop (SLT). IEEE, 1007-1013.

[18] David Snyder, Daniel Garcia-Romero, Gregory Sell, Daniel Povey, and Sanjeev Khudanpur. 2018. X-vectors: Robust dnn embeddings for speaker recognition. In IEEE ICASSP 2018. 5329-5333.

[19] Guy-Bart Stan, Jean-Jacques Embrechts, and Dominique Archambeau. 2002. Comparison of different impulse response measurement techniques. Fournal of the Audio Engineering Society 50, 4 (2002), 249-262.

[20] Christophe Veaux, Junichi Yamagishi, Kirsten MacDonald, et al. 2017. CSTR VCTK corpus: English multi-speaker corpus for CSTR voice cloning toolkit. University of Edinburgh. The Centre for Speech Technology Research (CSTR) (2017).

[21] Zhizheng Wu, Sheng Gao, Eng Siong Cling, and Haizhou Li. 2014. A study on replay attack and anti-spoofing for text-dependent speaker verification. In IEEE APSIPA 2014. 1-5.

[22] Zhizheng Wu, Tomi Kinnunen, Eng Siong Chng, Haizhou Li, and Eliathamby Ambikairajah. 2012. A study on spoofing attack in state-of-the-art speaker verification: the telephone speech case. In IEEE APSIPA ASC 2012. 1-5.

[23] Hong Yu, Zheng-Hua Tan, Yiming Zhang, Zhanyu Ma, and Jun Guo. 2017. DNN filter bank cepstral coefficients for spoofing detection. IEEE Access 5 (2017), 4779-4787.

[24] Xuejing Yuan, Yuxuan Chen, Yue Zhao, Yunhui Long, Xiaokang Liu, Kai Chen, Shengzhi Zhang, Heqing Huang, XiaoFeng Wang, and Carl A Gunter. 2018. Commandersong: A systematic approach for practical adversarial voice recognition. In 27th USENIX Security Symposium. 49-64. 